\title{
Evolution of Bonding During the Insertion of Anionic Ibuprofen into Model Cell Membranes
}

\author{
Natalia Rojas-Valencia, ${ }^{\dagger}$ Sara Gómez, ${ }^{\ddagger}$ Sebastian Montillo ${ }^{\dagger}$ \\ Marcela Manrique-Moreno, ${ }^{\dagger}$ Chiara Cappelli, ${ }^{\ddagger}$ Cacier Hadad, ${ }^{\dagger}$ Albeiro Restrepo ${ }^{\dagger}$ \\ ${ }^{\dagger}$ Instituto de Química, Universidad de Antioquia UdeA, Calle 70 No. 52-21, Medellín, \\ Colombia \\ ${ }^{\ddagger}$ Scuola Normale Superiore, Classe di Scienze, Piazza dei Cavalieri 7, 56126, Pisa, Italy
}

Supplementary information 


\section{Orbital interaction energies}

\begin{tabular}{|c|c|c|c|c|c|c|}
\hline \multirow[t]{2}{*}{ Label } & \multirow[t]{2}{*}{ Fragments } & \multirow{2}{*}{$\begin{array}{l}\text { Orbitals } \\
\text { involved }\end{array}$} & \multicolumn{4}{|c|}{$\begin{array}{c}-E_{i j}^{(2)} \\
\text { Level of theory }\end{array}$} \\
\hline & & & CAM-B3LYP & B3LYP & M062X & PBE0 \\
\hline & Intramolecular & & & & & \\
\hline 1 & $-\mathrm{CO}_{2}^{-}$in $\mathrm{Ibu}^{-}$ & $n_{\mathrm{O}} \rightarrow \pi_{\mathrm{C}=\mathrm{O}}^{*}$ & 124.56 & 95.05 & 122.95 & 100.05 \\
\hline 2 & $-\mathrm{COOR}$ in DMPC & $n_{\mathrm{O}} \rightarrow \pi_{\mathrm{C}=\mathrm{O}}^{*}$ & 76.92 & 61.88 & 74.77 & 64.40 \\
\hline 3 & -Non polar Ibu ${ }^{-}$ & $\pi_{\mathrm{C}=\mathrm{C}} \rightarrow \pi_{\mathrm{C}=\mathrm{C}}^{*}$ & 38.89 & 26.81 & 37.07 & 28.55 \\
\hline 4 & $-\mathrm{PO}_{2}^{-}$in $\mathrm{DMPC}$ & $n_{\mathrm{O}} \rightarrow \sigma_{\mathrm{P}=\mathrm{O}}^{*}$ & 31.81 & 25.47 & 31.46 & 26.73 \\
\hline & Intermolecular & & & & & \\
\hline 5 & $-\mathrm{CO}_{2}^{-}$in $\mathrm{Ibu}^{-} \rightarrow$ water & $n_{\mathrm{O}} \rightarrow \sigma_{\mathrm{O}-\mathrm{H}}^{*}$ & 29.19 & 24.46 & 27.37 & 24.82 \\
\hline 6 & water $\leftrightarrow$ water & $n_{\mathrm{O}} \rightarrow \sigma_{\mathrm{O}-\mathrm{H}}^{*}$ & 21.25 & 18.70 & 19.66 & 19.02 \\
\hline 7 & $-\mathrm{PO}_{2}^{-}$in $\mathrm{DMPC} \rightarrow$ water & $n_{\mathrm{O}} \rightarrow \sigma_{\mathrm{O}-\mathrm{H}}^{*}$ & 17.18 & 16.19 & 15.95 & 14.54 \\
\hline 8 & $-\mathrm{COOR}$ in $\mathrm{DMPC} \rightarrow$ water & $n_{\mathrm{O}} \rightarrow \sigma_{\mathrm{O}-\mathrm{H}}^{*}$ & 6.95 & 6.10 & 6.90 & 6.11 \\
\hline 9 & -Choline in DMPC $\rightarrow$ water & $n_{\mathrm{O}} \rightarrow \sigma_{\mathrm{C}-\mathrm{H}}^{*}$ & 5.08 & 4.60 & 4.86 & 4.78 \\
\hline 10 & $-\mathrm{CO}_{2}^{-}$in $\mathrm{Ibu}^{-} \rightarrow$ Choline in DMPC & $n_{\mathrm{O}} \rightarrow \sigma_{\mathrm{C}-\mathrm{H}}^{*}$ & 5.02 & 4.50 & 4.47 & 4.58 \\
\hline 11 & $-\mathrm{CO}_{2}^{-}$in $\mathrm{Ibu}^{-} \rightarrow$ Choline in DMPC & $\pi_{\mathrm{C}=\mathrm{O}} \rightarrow \sigma_{\mathrm{C}-\mathrm{H}}^{*}$ & 1.23 & 1.17 & 1.18 & 1.19 \\
\hline 12 & Non-polar $\mathrm{Ibu}^{-} \rightarrow$ Non-polar DMPC & $\sigma_{\mathrm{C}-\mathrm{H}} \rightarrow \sigma_{\mathrm{C}-\mathrm{H}}^{*}$ & 0.95 & 0.89 & 0.89 & 0.89 \\
\hline 13 & Non-polar Ibu ${ }^{-} \rightarrow$ Non-polar DMPC & $\pi_{\mathrm{C}=\mathrm{C}} \rightarrow \sigma_{\mathrm{C}-\mathrm{H}}^{*}$ & 0.17 & 0.16 & 0.16 & 0.16 \\
\hline 14 & Choline in DMPC $\rightarrow$ Non-polar $\mathrm{Ibu}^{-}$ & $\sigma_{\mathrm{C}-\mathrm{H}} \rightarrow \pi_{\mathrm{C}=\mathrm{C}}^{*}$ & 0.16 & 0.14 & 0.16 & 0.14 \\
\hline 15 & Non-polar $\mathrm{Ibu}^{-} \rightarrow$ Choline in DMPC & $\pi_{\mathrm{C}=\mathrm{C}} \rightarrow \sigma_{\mathrm{C}-\mathrm{H}}^{*}$ & 0.27 & 0.26 & 0.27 & 0.26 \\
\hline 16 & Non-polar $\mathrm{Ibu}^{-} \rightarrow$ water & $\pi_{\mathrm{C}=\mathrm{C}} \rightarrow \sigma_{\mathrm{O}-\mathrm{H}}^{*}$ & $\mathrm{~N} / \mathrm{A}$ & $\mathrm{N} / \mathrm{A}$ & $\mathrm{N} / \mathrm{A}$ & $\mathrm{N} / \mathrm{A}$ \\
\hline 17 & Non-polar $\mathrm{Ibu}^{-} \rightarrow$ water & $\sigma_{\mathrm{C}-\mathrm{H}} \rightarrow \sigma_{\mathrm{O}-\mathrm{H}}^{*}$ & 0.09 & 0.09 & 0.01 & 0.09 \\
\hline 18 & water $\rightarrow$ Non-polar $\mathrm{Ibu}^{-}$ & $n_{\mathrm{O}} \rightarrow \sigma_{\mathrm{C}-\mathrm{H}}^{*}$ & 0.06 & 0.05 & 0.06 & 0.05 \\
\hline 19 & water $\rightarrow$ Non-polar $\mathrm{Ibu}^{-}$ & $n_{\mathrm{O}} \rightarrow \sigma_{\mathrm{C}-\mathrm{H}}^{*}$ & $\mathrm{~N} / \mathrm{A}$ & $\mathrm{N} / \mathrm{A}$ & $\mathrm{N} / \mathrm{A}$ & $\mathrm{N} / \mathrm{A}$ \\
\hline
\end{tabular}

Table S1: Largest NBO energies, $-E_{i j}^{(2)}$, in $\mathrm{kcal} / \mathrm{mol}$ and electron densities, $\rho\left(\mathbf{r}_{c}\right)$, in a.u. at the bond critical points for all interaction types found in a randomly chosen frame (same frame as in Figure 6,7 and 9) of the MD simulations at the free energy minimum in Figure 1. Figure 7 shows the specific involved orbitals in each case and Figure 9 contains the molecular graphs. 


\section{Geometries}

Cartesian coordinates for the three randomly chosen configurations used to study bonding interactions. The frames were drawn from MD simulations at the three equilibrium positions of the free energy profile for the tertiary $\mathrm{Ibu}^{-} /$membrane/water system in Figure 1.

\begin{tabular}{|c|c|c|c|}
\hline 6 & -0.006794 & 0.969782 & -1.227890 \\
\hline 6 & -1.315848 & 0.837365 & -0.814438 \\
\hline 6 & -1.786150 & -0.378334 & -0.174288 \\
\hline 6 & -0.859388 & -1.436432 & -0.020531 \\
\hline 6 & 0.408737 & -1.280655 & -0.487641 \\
\hline 6 & 0.891803 & -0.096693 & -1.097298 \\
\hline 1 & 0.241906 & 1.963999 & -1.540110 \\
\hline 1 & -2.067717 & 1.584615 & -1.046842 \\
\hline 1 & -1.252743 & -2.368753 & 0.367476 \\
\hline 1 & 1.044758 & -2.155398 & -0.487022 \\
\hline 6 & -3.205074 & -0.534222 & 0.316480 \\
\hline 1 & -3.265461 & -1.530757 & 0.793421 \\
\hline 6 & 2.355815 & -0.051334 & -1.525213 \\
\hline 1 & 2.548819 & -0.897609 & -2.217937 \\
\hline 1 & 2.511063 & 0.882165 & -2.120593 \\
\hline 6 & -3.518995 & 0.490521 & 1.363645 \\
\hline 1 & -3.423383 & 1.569634 & 1.098931 \\
\hline 1 & -2.813430 & 0.306775 & 2.195400 \\
\hline 1 & -4.539981 & 0.316050 & 1.770151 \\
\hline 6 & -4.145416 & -0.558018 & -0.875699 \\
\hline 8 & -4.746350 & 0.480923 & -1.336652 \\
\hline 8 & -4.316805 & -1.686487 & -1.381648 \\
\hline 6 & 3.359770 & -0.051504 & -0.371491 \\
\hline 6 & 4.583111 & -0.920714 & -0.866781 \\
\hline 6 & 3.944424 & 1.338200 & 0.085786 \\
\hline 1 & 2.947013 & -0.580999 & 0.516070 \\
\hline 1 & 5.530094 & -0.663814 & -0.349746 \\
\hline 1 & 4.772368 & -0.684632 & -1.933387 \\
\hline 1 & 4.438065 & -2.010907 & -0.684733 \\
\hline 1 & 3.149612 & 2.106635 & 0.247598 \\
\hline 1 & 4.568034 & 1.749891 & -0.730621 \\
\hline 1 & 4.641107 & 1.296752 & 0.954586 \\
\hline 8 & -0.613126 & -4.637239 & 1.411633 \\
\hline 1 & -1.260768 & -5.329110 & 1.257771 \\
\hline
\end{tabular}




\begin{tabular}{|c|c|c|c|}
\hline 1 & -0.094892 & -4.606820 & 0.606519 \\
\hline 8 & 1.038442 & 4.895055 & -0.879021 \\
\hline 1 & 0.830139 & 5.798261 & -1.107776 \\
\hline 1 & 1.528373 & 4.957149 & -0.055303 \\
\hline 8 & 3.145245 & -5.007397 & -1.491492 \\
\hline 1 & 2.864424 & -5.880847 & -1.227058 \\
\hline 1 & 3.225467 & -4.518300 & -0.665086 \\
\hline 8 & -4.486973 & -2.919315 & -5.063098 \\
\hline 1 & -4.629020 & -3.122316 & -4.139241 \\
\hline 1 & -3.536776 & -2.835870 & -5.147730 \\
\hline 8 & 4.483486 & -1.200666 & 2.973115 \\
\hline 1 & 4.539787 & -0.246124 & 2.926430 \\
\hline 1 & 3.558255 & -1.379624 & 3.174168 \\
\hline 8 & 7.135343 & 3.191604 & 1.476048 \\
\hline 1 & 7.504390 & 3.889360 & 2.018303 \\
\hline 1 & 7.720168 & 2.449376 & 1.603232 \\
\hline 8 & 3.658889 & 3.980195 & -1.895183 \\
\hline 1 & 3.772528 & 4.038948 & -2.835945 \\
\hline 1 & 2.714822 & 3.828410 & -1.776025 \\
\hline 8 & -6.203318 & -2.925960 & 3.207815 \\
\hline 1 & -6.638779 & -2.541649 & 2.458630 \\
\hline 1 & -5.275942 & -2.946891 & 2.981463 \\
\hline 8 & 1.902238 & -0.906860 & 3.674070 \\
\hline 1 & 1.617251 & -1.813588 & 3.679322 \\
\hline 1 & 1.286341 & -0.454822 & 3.093869 \\
\hline 8 & 6.301610 & 4.017244 & -0.955847 \\
\hline 1 & 6.498394 & 3.592173 & -0.126213 \\
\hline 1 & 5.353190 & 3.920550 & -1.062843 \\
\hline 8 & -3.246435 & -3.183494 & 3.181013 \\
\hline 1 & -2.387142 & -2.979556 & 2.800716 \\
\hline 1 & -3.454040 & -4.056487 & 2.851192 \\
\hline 8 & -7.131292 & -1.781009 & 0.865690 \\
\hline 1 & -7.754209 & -1.088190 & 1.070331 \\
\hline 1 & -6.509790 & -1.382582 & 0.272486 \\
\hline 8 & 0.825555 & 3.146640 & 2.489929 \\
\hline 1 & 1.295916 & 2.828296 & 3.250533 \\
\hline 1 & 0.162816 & 2.477620 & 2.320258 \\
\hline 8 & -5.469707 & 0.750470 & 4.405429 \\
\hline 1 & -6.206619 & 0.689640 & 5.021189 \\
\hline 1 & -5.076132 & -0.124259 & 4.428848 \\
\hline 8 & -4.040607 & 4.125984 & 0.968138 \\
\hline 1 & -3.545576 & 3.659064 & 1.635916 \\
\hline
\end{tabular}




\begin{tabular}{|c|c|c|c|}
\hline 1 & -4.728515 & 3.510500 & 0.697469 \\
\hline 8 & 0.610403 & -3.426024 & 3.530981 \\
\hline 1 & 0.308345 & -3.658382 & 2.661285 \\
\hline 1 & 0.884917 & -4.254793 & 3.924282 \\
\hline 8 & -5.578422 & -3.430858 & -2.774089 \\
\hline 1 & -5.093375 & -2.777970 & -2.274623 \\
\hline 1 & -6.108811 & -3.897899 & -2.133027 \\
\hline 8 & 7.827816 & -1.211140 & 0.991563 \\
\hline 1 & 8.087346 & -0.370096 & 1.364778 \\
\hline 1 & 7.921997 & -1.084345 & 0.041219 \\
\hline 8 & -2.005522 & 2.824334 & 5.543455 \\
\hline 1 & -1.656356 & 2.132961 & 4.976105 \\
\hline 1 & -1.824384 & 2.520424 & 6.430319 \\
\hline 8 & 5.702862 & -2.803322 & -3.265541 \\
\hline 1 & 4.867981 & -3.235452 & -3.428136 \\
\hline 1 & 6.034276 & -2.596007 & -4.136279 \\
\hline 8 & 2.586372 & 2.226678 & -4.373284 \\
\hline 1 & 2.839357 & 1.315591 & -4.489552 \\
\hline 1 & 3.357755 & 2.723564 & -4.644710 \\
\hline 8 & -6.073825 & 3.512256 & -3.853601 \\
\hline 1 & -5.213858 & 3.108773 & -3.695623 \\
\hline 1 & -6.628506 & 2.795963 & -4.150507 \\
\hline 8 & -3.920787 & 1.830310 & -3.773721 \\
\hline 1 & -3.921599 & 1.168756 & -3.084557 \\
\hline 1 & -3.208657 & 2.416516 & -3.530549 \\
\hline 8 & -1.806945 & -0.322779 & 4.385206 \\
\hline 1 & -2.759213 & -0.446040 & 4.452219 \\
\hline 1 & -1.428236 & -1.058589 & 4.851322 \\
\hline 8 & -1.889369 & 4.151379 & -0.840655 \\
\hline 1 & -1.142074 & 3.991733 & -0.266927 \\
\hline 1 & -2.570929 & 4.465157 & -0.254977 \\
\hline 8 & -6.083414 & 2.302074 & 0.023262 \\
\hline 1 & -6.801924 & 2.429228 & -0.599615 \\
\hline 1 & -5.742320 & 1.437160 & -0.173658 \\
\hline 8 & -4.755501 & -3.818044 & 0.643422 \\
\hline 1 & -5.676546 & -3.636875 & 0.450151 \\
\hline 1 & -4.267081 & -3.203927 & 0.077908 \\
\hline 8 & -1.872694 & 3.520581 & -3.412894 \\
\hline 1 & -1.799942 & 3.841622 & -2.512317 \\
\hline 1 & -2.120032 & 4.288090 & -3.920688 \\
\hline 8 & -6.195527 & 2.833525 & 2.751777 \\
\hline 1 & -6.130703 & 1.973482 & 3.160951 \\
\hline
\end{tabular}




$\begin{array}{cccc}1 & -6.173823 & 2.662640 & 1.807252 \\ 8 & 0.003451 & 1.135151 & -4.395342 \\ 1 & 0.826773 & 1.543833 & -4.167045 \\ 1 & -0.667743 & 1.657909 & -3.953955 \\ 8 & 3.337233 & -4.091264 & 1.211680 \\ 1 & 3.344397 & -3.281338 & 1.730302 \\ 1 & 3.461185 & -4.794173 & 1.854762 \\ 8 & -2.332860 & -2.098988 & -3.100661 \\ 1 & -3.192322 & -1.777164 & -2.858474 \\ 1 & -1.761008 & -1.849476 & -2.375245 \\ 8 & 7.831916 & -0.997233 & -1.745649 \\ 1 & 7.285449 & -1.425103 & -2.411533 \\ 1 & 8.598613 & -1.559705 & -1.671476 \\ 8 & -0.028759 & -4.558156 & -1.321838 \\ 1 & 0.638365 & -4.201768 & -1.899188 \\ 1 & -0.700250 & -4.913742 & -1.909339 \\ 8 & -7.392693 & 0.115785 & -1.937102 \\ 1 & -7.598112 & 0.521879 & -2.779593 \\ 1 & -6.448504 & 0.242205 & -1.840027 \\ 8 & 6.458193 & -3.440302 & 1.740409 \\ 1 & 6.937848 & -2.829697 & 1.178187 \\ 1 & 5.867160 & -2.883676 & 2.249081 \\ 8 & 7.410211 & -4.543669 & -1.275804 \\ 1 & 7.080171 & -5.145331 & -0.609489 \\ 1 & 6.620456 & -4.211543 & -1.717822 \\ 8 & 5.659682 & 3.674333 & 3.924455 \\ 1 & 5.432175 & 3.482325 & 3.022915 \\ 1 & 4.915222 & 4.168897 & 4.262221 \\ 8 & 2.428195 & 5.319250 & 1.784725 \\ 1 & 1.842151 & 4.601236 & 2.040280 \\ 1 & 2.857421 & 5.576581 & 2.594383 \\ 8 & 8.230228 & 1.612098 & -1.485571 \\ 1 & 8.594823 & 0.737786 & -1.629962 \\ 1 & 7.292594 & 1.454938 & -1.355023 \\ 8 & -0.215531 & 0.619925 & 2.368473 \\ 1 & -0.653052 & 0.227919 & 3.124189 \\ 1 & -0.614837 & 0.217476 & 1.605133\end{array}$

Top of the barrier, Energy $=-6744.22871897 \mathrm{H}$
$\begin{array}{cccc}6 & -0.858354 & 0.957532 & -0.921211 \\ 6 & -1.265141 & 2.307937 & -0.819569\end{array}$




$\begin{array}{cccc}6 & -1.711036 & 2.786945 & 0.412799 \\ 6 & -1.867952 & 1.873089 & 1.467903 \\ 6 & -1.479133 & 0.543722 & 1.345415 \\ 6 & -0.871769 & 0.072423 & 0.202311 \\ 1 & -0.498981 & 0.590481 & -1.870066 \\ 1 & -0.958563 & 2.974598 & -1.619926 \\ 1 & -2.208477 & 2.243146 & 2.422733 \\ 1 & -1.603278 & -0.184922 & 2.137038 \\ 6 & -1.960502 & 4.287819 & 0.511498 \\ 1 & -1.985104 & 4.802686 & -0.473188 \\ 6 & -0.330098 & -1.327942 & 0.135459 \\ 1 & 0.535317 & -1.394229 & -0.552699 \\ 1 & -0.022569 & -1.541305 & 1.180544 \\ 6 & -3.281546 & 4.568421 & 1.290603 \\ 1 & -4.173058 & 4.220863 & 0.729701 \\ 1 & -3.392523 & 5.668627 & 1.354876 \\ 1 & -3.310019 & 4.118359 & 2.309687 \\ 6 & -0.634169 & 4.724810 & 1.162020 \\ 8 & -0.528838 & 4.555586 & 2.387569 \\ 8 & 0.280095 & 5.214922 & 0.408023 \\ 6 & -1.341321 & -2.428573 & -0.237958 \\ 6 & -0.613733 & -3.769758 & -0.184674 \\ 6 & -2.485397 & -2.563287 & 0.727413 \\ 1 & -1.692135 & -2.238129 & -1.268861 \\ 1 & -0.365835 & -4.107853 & 0.843498 \\ 1 & 0.325394 & -3.755079 & -0.777906 \\ 1 & -1.382618 & -4.465762 & -0.588897 \\ 1 & -2.990147 & -1.580499 & 0.887894 \\ 1 & -2.165580 & -2.978960 & 1.701647 \\ 1 & -3.209195 & -3.181712 & 0.141948 \\ 7 & 3.302685 & 2.607400 & 1.992599 \\ 6 & 4.753661 & 2.172217 & 1.818575 \\ 1 & 5.372305 & 2.592089 & 2.601319 \\ 1 & 5.107111 & 2.447972 & 0.832759 \\ 1 & 4.802519 & 1.093425 & 1.839052 \\ 6 & 3.306142 & 3.976050 & 2.564950 \\ 1 & 2.265824 & 4.227160 & 2.707720 \\ 1 & 3.816626 & 4.743972 & 0.631153 \\ 1 & 3.839213 & 4.021921 & \\ 6 & 2.640460 & 2.679672 & \\ 1 & 1.724222 & 3.249371 & \\ 1 & 2.539069 & 1.633554 & \\ & & & \\ 1 & & 0.372780 \\ 1 & \end{array}$




$\begin{array}{lccc}1 & 3.260471 & 3.227442 & -0.060464 \\ 6 & 2.456707 & 1.835713 & 2.873689 \\ 1 & 1.413032 & 2.105041 & 2.817067 \\ 1 & 2.804408 & 2.046130 & 3.880083 \\ 6 & 2.697158 & 0.250681 & 2.611478 \\ 1 & 3.749769 & -0.051518 & 2.790327 \\ 1 & 2.323251 & -0.059295 & 1.603952 \\ 15 & 2.545969 & -1.140282 & 4.859157 \\ 8 & 1.563294 & -1.352756 & 5.923538 \\ 8 & 3.731759 & -0.295677 & 5.245864 \\ 8 & 3.095977 & -2.495010 & 4.207543 \\ 8 & 1.925327 & -0.443798 & 3.554545 \\ 6 & 2.192155 & -3.314626 & 3.487865 \\ 1 & 2.691757 & -3.961933 & 2.739741 \\ 1 & 1.421268 & -2.774611 & 2.903214 \\ 6 & 1.360614 & -4.250865 & 4.372736 \\ 1 & 0.912285 & -3.723023 & 5.253523 \\ 8 & 2.261812 & -5.258235 & 4.881901 \\ 6 & 1.971053 & -5.848250 & 5.995427 \\ 8 & 1.089539 & -5.485354 & 6.852889 \\ 6 & 2.736829 & -7.133269 & 5.966937 \\ 1 & 2.787725 & -7.399989 & 7.045070 \\ 1 & 3.742023 & -6.927922 & 5.537964 \\ 6 & 0.063291 & -4.846021 & 3.710284 \\ 1 & -0.490212 & -3.914898 & 3.448090 \\ 1 & -0.511354 & -5.418583 & 4.472095 \\ 8 & 0.428068 & -5.524899 & 2.499638 \\ 6 & -0.469548 & -6.478441 & 2.195924 \\ 8 & -1.529996 & -6.615976 & 2.798530 \\ 6 & 0.084458 & -7.258371 & 1.075171 \\ 1 & 1.195190 & -7.220945 & 1.134116 \\ 1 & -0.253318 & -6.790102 & 0.131121 \\ 1 & 2.204826 & -7.881862 & 5.410987 \\ 1 & -0.173375 & -8.295473 & 1.171826 \\ 7 & -6.294700 & 0.217419 & 0.533049 \\ 6 & -4.942240 & 0.636941 & 1.143419 \\ 1 & -4.831594 & 0.007303 & 2.014862 \\ 1 & -4.859745 & 1.657701 & 1.507622 \\ 1 & -4.100074 & 0.431649 & 0.498119 \\ 6 & -6.550260 & -1.253795 & 0.506247 \\ 1 & -6.612170 & -1.760588 & 1.458522 \\ 1 & -5.869069 & -1.876672 & -057139\end{array}$




$\begin{array}{lccc}1 & -7.550039 & -1.399045 & 0.118116 \\ 6 & -7.346502 & 0.848740 & 1.424422 \\ 1 & -8.352309 & 0.674562 & 1.069859 \\ 1 & -7.243754 & 1.921717 & 1.497655 \\ 1 & -7.370637 & 0.464988 & 2.429933 \\ 6 & -6.238631 & 0.768912 & -0.858394 \\ 1 & -6.301231 & 1.843010 & -0.766225 \\ 1 & -7.081205 & 0.530869 & -1.494872 \\ 6 & -5.014035 & 0.381610 & -1.681232 \\ 1 & -4.887176 & -0.707702 & -1.834551 \\ 1 & -4.093319 & 0.795853 & -1.212527 \\ 15 & -6.115912 & 0.452910 & -3.998296 \\ 8 & -5.908075 & 1.248423 & -5.187944 \\ 8 & -7.494497 & 0.457185 & -3.467651 \\ 8 & -5.666308 & -0.981275 & -4.259775 \\ 8 & -5.006380 & 0.968418 & -2.937734 \\ 6 & -4.761522 & -1.261711 & -5.399475 \\ 1 & -3.942333 & -0.533217 & -5.571893 \\ 1 & -5.375130 & -1.308711 & -6.318755 \\ 6 & -3.998040 & -2.651543 & -5.167180 \\ 1 & -3.465956 & -2.907383 & -6.114298 \\ 8 & -4.925401 & -3.608833 & -4.806213 \\ 6 & -5.531280 & -4.228199 & -5.830077 \\ 8 & -5.580359 & -3.859480 & -6.998725 \\ 6 & -6.328164 & -5.387229 & -5.410999 \\ 1 & -6.851513 & -5.606292 & -6.361061 \\ 1 & -7.075630 & -5.091765 & -4.641196 \\ 6 & -2.835275 & -2.562254 & -4.160499 \\ 1 & -3.295489 & -2.406344 & -3.155514 \\ 1 & -2.181309 & -1.693260 & -4.384503 \\ 8 & -1.963235 & -3.749400 & -4.037898 \\ 6 & -2.422122 & -4.732586 & -3.233804 \\ 8 & -3.439568 & -4.625168 & -2.555377 \\ 6 & -1.451532 & -5.933067 & -3.247988 \\ 1 & -1.252367 & -6.124268 & -2.173476 \\ 1 & -0.420626 & -5.678314 & -3.582104 \\ 1 & -5.725333 & -6.219166 & -5.120011 \\ 1 & -1.832881 & -6.812768 & -3.711127 \\ 7 & 9.566604 & 2.940793 & -3.607720 \\ 6 & 9.822574 & 2.732083 & -5.046407 \\ 1 & 8.882838 & 2.628910 & \\ 1 & 10.279383 & 1.771645 & \\ & & & -5780 \\ 1 & \end{array}$




\begin{tabular}{|c|c|c|c|}
\hline 1 & 10.508609 & 3.463949 & -5.436445 \\
\hline 6 & 10.876668 & 3.392999 & -2.947007 \\
\hline 1 & 10.737538 & 3.336094 & -1.877099 \\
\hline 1 & 11.239450 & 4.372246 & -3.211705 \\
\hline . & 11.645149 & 2.725609 & -3.294756 \\
\hline 6 & 8.508950 & 4.045283 & -3.471447 \\
\hline 1 & 8.884156 & 4.993159 & -3.826485 \\
\hline 1 & 8.243550 & 4.101268 & -2.428217 \\
\hline 1 & 7.633844 & 3.853449 & -4.062030 \\
\hline 6 & 9.205172 & 1.632260 & -2.947335 \\
\hline 1 & 8.877399 & 1.890926 & -1.943215 \\
\hline 1 & 10.021903 & 0.934825 & -2.829713 \\
\hline 6 & 7.978392 & 0.869550 & -3.580645 \\
\hline 1 & 7.908538 & -0.083758 & -3.014584 \\
\hline 1 & 8.431185 & 0.547397 & -4.545526 \\
\hline 15 & 5.718329 & 1.687542 & -2.620277 \\
\hline 8 & 4.414506 & 2.147702 & -3.190714 \\
\hline 8 & 6.205129 & 2.386178 & -1.394580 \\
\hline 8 & 5.582552 & 0.134813 & -2.314502 \\
\hline 8 & 6.822984 & 1.743169 & -3.756210 \\
\hline 6 & 5.566001 & -0.292889 & -0.976109 \\
\hline 1 & 4.685072 & 0.149085 & -0.461498 \\
\hline 1 & 6.537903 & -0.146699 & -0.467056 \\
\hline 6 & 5.094914 & -1.791534 & -0.844810 \\
\hline 1 & 4.914121 & -1.994209 & 0.229032 \\
\hline 8 & 6.031582 & -2.742755 & -1.412906 \\
\hline 6 & 7.193770 & -2.971869 & -0.746254 \\
\hline 8 & 7.599172 & -2.370732 & 0.249730 \\
\hline 6 & 7.862142 & -4.164295 & -1.451234 \\
\hline 1 & 8.338675 & -4.707135 & -0.606106 \\
\hline 1 & 8.712471 & -3.754769 & -2.029965 \\
\hline 6 & 3.649291 & -1.961415 & -1.554681 \\
\hline 1 & 3.547088 & -1.550243 & -2.577125 \\
\hline 1 & 2.941348 & -1.526381 & -0.812412 \\
\hline 8 & 3.305925 & -3.381724 & -1.464014 \\
\hline 6 & 3.614432 & -4.187772 & -2.466666 \\
\hline 8 & 4.220675 & -3.859037 & -3.540166 \\
\hline 6 & 3.113808 & -5.553420 & -2.041362 \\
\hline 1 & 2.274534 & -5.457264 & -1.316811 \\
\hline 1 & 3.920686 & -6.097379 & -1.501310 \\
\hline 1 & 7.268132 & -4.799434 & -2.069499 \\
\hline 1 & 2.719491 & -6.149669 & -2.84048 \\
\hline
\end{tabular}




\begin{tabular}{|c|c|c|c|}
\hline 3 & -3.887027 & -5.438263 & 3.783366 \\
\hline 1 & -3.038278 & -5.835150 & 3.581855 \\
\hline 1 & -4.527853 & -6.104392 & 3.529172 \\
\hline 8 & -4.969227 & 4.754378 & 4.746388 \\
\hline 1 & -5.408340 & 4.416064 & 5.531833 \\
\hline 1 & -4.264170 & 4.119674 & 4.582240 \\
\hline 8 & 1.066121 & 4.700773 & -2.515402 \\
\hline 1 & 0.710490 & 4.888055 & -1.655256 \\
\hline 1 & 0.293868 & 4.586899 & -3.067995 \\
\hline 8 & 0.905764 & -0.728932 & -4.566033 \\
\hline 1 & 0.314093 & -1.117242 & -5.215449 \\
\hline 1 & 1.576434 & -1.410218 & -4.430936 \\
\hline 8 & -1.837952 & 5.756461 & 4.372971 \\
\hline 1 & -2.165089 & 4.925615 & 4.718333 \\
\hline 1 & -1.441234 & 5.531988 & 3.535817 \\
\hline 8 & -6.725382 & 6.021676 & 3.288864 \\
\hline 1 & -5.971375 & 5.798930 & 3.834078 \\
\hline 1 & -6.351798 & 6.361489 & 2.475069 \\
\hline 8 & 0.932638 & 4.112175 & 4.460459 \\
\hline 1 & 0.796083 & 3.191356 & 4.685059 \\
\hline 1 & 0.349980 & 4.268051 & 3.709651 \\
\hline 8 & -5.857304 & 7.571764 & 0.574224 \\
\hline 1 & -5.426099 & 7.113991 & -0.153928 \\
\hline 1 & -6.660092 & 7.919052 & 0.187154 \\
\hline 8 & -0.063813 & 1.386887 & 5.025162 \\
\hline 1 & -0.665580 & 0.769410 & 4.623295 \\
\hline 1 & 0.780564 & 0.948678 & 5.025575 \\
\hline 8 & 2.211608 & 0.902026 & -2.443430 \\
\hline 1 & 2.840151 & 1.515359 & -2.826780 \\
\hline 1 & 1.517811 & 0.836993 & -3.100709 \\
\hline 8 & -3.407831 & 7.963953 & 4.164262 \\
\hline 1 & -3.985922 & 7.925973 & 3.396167 \\
\hline 1 & -2.804801 & 7.237201 & 4.033544 \\
\hline 8 & -1.271333 & 4.890384 & -3.772724 \\
\hline 1 & -1.848877 & 4.140294 & -3.849589 \\
\hline 1 & -1.625827 & 5.396249 & -3.042975 \\
\hline 8 & 2.724818 & 6.887397 & -0.011446 \\
\hline 1 & 2.866703 & 7.259698 & -0.880561 \\
\hline 1 & 1.814358 & 6.611793 & -0.013274 \\
\hline 8 & -5.226565 & -4.086993 & 1.638906 \\
\hline 1 & -6.107361 & -4.085331 & 2.006461 \\
\hline 1 & -4.670116 & -4.358207 & 2.363066 \\
\hline
\end{tabular}




$\begin{array}{cccc}8 & -4.967714 & 6.553639 & -1.853336 \\ 1 & -5.236433 & 7.283585 & -2.410532 \\ 1 & -5.593585 & 5.852009 & -2.069900 \\ 8 & -2.531822 & 3.258479 & 4.973409 \\ 1 & -2.733192 & 2.963944 & 5.857151 \\ 1 & -1.777831 & 2.717510 & 4.708170 \\ 8 & -1.881863 & -0.543298 & 4.769082 \\ 1 & -1.891201 & -1.467493 & 4.995746 \\ 1 & -2.610766 & -0.158236 & 5.267909 \\ 8 & 0.972124 & 6.933019 & 2.715930 \\ 1 & 0.403662 & 6.217114 & 2.425879 \\ 1 & 1.651721 & 6.987698 & 2.050980 \\ 8 & -3.148519 & 2.561034 & -3.507585 \\ 1 & -3.760793 & 1.932486 & -3.134425 \\ 1 & -2.594038 & 2.039024 & -4.090214 \\ 8 & -2.411405 & -3.253164 & 5.359431 \\ 1 & -3.159160 & -2.886888 & 5.826768 \\ 1 & -2.787034 & -3.960800 & 4.825153 \\ 8 & -5.640164 & -3.812616 & -1.344741 \\ 1 & -5.046102 & -4.141145 & -2.015680 \\ 1 & -5.193761 & -3.992729 & -0.520130 \\ 8 & -2.311973 & 6.970005 & -2.072153 \\ 1 & -3.259838 & 7.093382 & -2.022854 \\ 1 & -2.054095 & 6.753256 & -1.169978\end{array}$

Energy minimum, Energy=-7006.27341431 Hartrees

$\begin{array}{cccc}6 & 3.293878 & 0.362991 & 0.167615 \\ 6 & 1.991471 & 0.814494 & -0.064078 \\ 6 & 1.009016 & 0.365105 & 0.739807 \\ 6 & 1.246639 & -0.544117 & 1.804584 \\ 6 & 2.503707 & -1.057292 & 1.997022 \\ 6 & 3.549560 & -0.594904 & 1.152991 \\ 1 & 4.114865 & 0.663845 & -0.466077 \\ 1 & 1.739159 & 1.508731 & -0.848025 \\ 1 & 0.360144 & -0.925458 & 2.288843 \\ 1 & 2.841190 & -1.764809 & 2.746770 \\ 6 & -0.389364 & 0.853782 & 0.552915 \\ 1 & -1.114030 & 0.405483 & 1.258597 \\ 6 & 4.839213 & -1.359598 & 1.190908 \\ 1 & 4.546137 & -2.431806 & 1.158100 \\ 1 & 5.288502 & -1.324239 & 0.173220\end{array}$




\begin{tabular}{|c|c|c|c|}
\hline 6 & -0.373930 & 2.351791 & 0.927787 \\
\hline 1 & -0.170135 & 2.559375 & 2.006804 \\
\hline 1 & -1.323674 & 2.876828 & 0.738785 \\
\hline 1 & 0.334418 & 2.888707 & 0.269862 \\
\hline 6 & -0.818060 & 0.404008 & -0.907367 \\
\hline 8 & -0.437332 & 1.068247 & -1.921878 \\
\hline 8 & -1.582646 & -0.616728 & -0.970659 \\
\hline 6 & 5.838620 & -0.998905 & 2.320503 \\
\hline 6 & 7.212003 & -1.410080 & 1.798051 \\
\hline 6 & 5.754536 & 0.535391 & 2.681982 \\
\hline 1 & 5.615764 & -1.541717 & 3.259966 \\
\hline 1 & 7.249981 & -2.457094 & 1.404455 \\
\hline 1 & 8.036629 & -1.253595 & 2.530720 \\
\hline 1 & 7.442756 & -0.742489 & 0.943841 \\
\hline 1 & 5.733750 & 1.048069 & 1.694243 \\
\hline 1 & 6.618217 & 0.874775 & 3.306380 \\
\hline 1 & 4.806885 & 0.689902 & 3.235683 \\
\hline 7 & -7.199202 & -7.071619 & -1.767262 \\
\hline 6 & -6.286234 & -8.183007 & -1.445073 \\
\hline 1 & -5.422194 & -8.152516 & -2.085856 \\
\hline 1 & -6.859084 & -9.082781 & -1.642176 \\
\hline 1 & -6.002144 & -8.266870 & -0.400870 \\
\hline 6 & -8.043525 & -7.659379 & -2.887377 \\
\hline 1 & -8.609127 & -6.806390 & -3.235379 \\
\hline 1 & -8.747449 & -8.448876 & -2.655460 \\
\hline 1 & -7.403535 & -7.916828 & -3.711168 \\
\hline 6 & -8.202507 & -6.883994 & -0.628150 \\
\hline 1 & -7.710425 & -6.808448 & 0.325342 \\
\hline 1 & -8.830343 & -7.760751 & -0.555909 \\
\hline 1 & -8.848297 & -6.035375 & -0.802801 \\
\hline 6 & -6.479844 & -5.817071 & -2.220290 \\
\hline 1 & -7.168299 & -5.121302 & -2.678695 \\
\hline 1 & -5.777493 & -6.078970 & -2.989388 \\
\hline 6 & -5.704164 & -5.055572 & -1.090052 \\
\hline 1 & -5.193573 & -4.146967 & -1.466660 \\
\hline 1 & -4.970674 & -5.796215 & -0.692130 \\
\hline 15 & -7.503234 & -3.571590 & 0.014186 \\
\hline 8 & -8.800335 & -3.977123 & 0.549604 \\
\hline 8 & -7.504432 & -2.920395 & -1.341153 \\
\hline 8 & -6.889676 & -2.510514 & 1.077560 \\
\hline 8 & -6.482119 & -4.731646 & 0.028097 \\
\hline 6 & -6.086649 & -3.146953 & 2.084831 \\
\hline
\end{tabular}




\begin{tabular}{|c|c|c|c|}
\hline 1 & -5.187355 & -3.606072 & 1.640106 \\
\hline 1 & -6.657187 & -3.877074 & 2.696067 \\
\hline 6 & -5.570574 & -2.194203 & 3.160951 \\
\hline 1 & -4.904089 & -2.773607 & 3.844025 \\
\hline 8 & -6.693454 & -1.645562 & 3.911772 \\
\hline 6 & -7.343477 & -2.357558 & 4.759553 \\
\hline 8 & -7.069572 & -3.486807 & 5.055649 \\
\hline 6 & -8.459376 & -1.559389 & 5.275882 \\
\hline 1 & -9.206153 & -1.625374 & 4.456327 \\
\hline 1 & -8.210064 & -0.482352 & 5.422976 \\
\hline 6 & -4.725425 & -0.994638 & 2.677041 \\
\hline 1 & -5.272958 & -0.439733 & 1.887429 \\
\hline 1 & -3.901088 & -1.539644 & 2.160866 \\
\hline 8 & -4.234006 & -0.221571 & 3.755686 \\
\hline 6 & -3.978217 & 0.992411 & 3.374477 \\
\hline 8 & -4.056466 & 1.433071 & 2.245276 \\
\hline 6 & -3.630533 & 1.816356 & 4.531555 \\
\hline 1 & -4.389138 & 1.648869 & 5.324376 \\
\hline 1 & -3.672155 & 2.860827 & 4.153867 \\
\hline 1 & -8.809176 & -2.059950 & 6.160572 \\
\hline 6 & -2.198948 & 1.628414 & 5.017705 \\
\hline 1 & -1.891414 & 2.477925 & 5.661330 \\
\hline 1 & -1.484831 & 1.640417 & 4.171542 \\
\hline 6 & -1.936385 & 0.375155 & 5.883444 \\
\hline 1 & -2.467507 & -0.432898 & 5.333572 \\
\hline 1 & -2.312270 & 0.496999 & 6.923475 \\
\hline 6 & -0.481561 & -0.017261 & 5.986384 \\
\hline 1 & -0.098681 & 0.591050 & 6.835301 \\
\hline 1 & 0.007581 & 0.080962 & 4.999676 \\
\hline 6 & -0.376222 & -1.539859 & 6.506671 \\
\hline 1 & -0.479006 & -2.172368 & 5.601537 \\
\hline 1 & -1.281657 & -1.715183 & 7.129847 \\
\hline 6 & 0.970088 & -1.815028 & 7.121351 \\
\hline 1 & 0.923446 & -2.856869 & 7.497503 \\
\hline 1 & 1.151720 & -1.254078 & 8.060577 \\
\hline 6 & 2.125203 & -1.616145 & 6.058169 \\
\hline 1 & 2.130188 & -0.550048 & 5.773540 \\
\hline 1 & 1.782846 & -2.123626 & 5.136511 \\
\hline 6 & 3.515823 & -2.096499 & 6.444736 \\
\hline 1 & 4.143164 & -2.074184 & 5.524657 \\
\hline 1 & 3.410365 & -3.133004 & 6.824390 \\
\hline 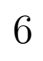 & 4.168659 & -1.201119 & 7.506527 \\
\hline
\end{tabular}




$\begin{array}{lccc}1 & 3.618949 & -1.180664 & 8.465902 \\ 1 & 4.131453 & -0.111757 & 7.277382 \\ 1 & 5.160187 & -1.464024 & 7.826454 \\ 7 & 1.420211 & -2.679644 & -2.448708 \\ 6 & 0.623118 & -2.552061 & -1.152843 \\ 1 & 1.386980 & -2.679260 & -0.408709 \\ 1 & -0.113833 & -3.329686 & -1.151075 \\ 1 & 0.130399 & -1.600019 & -1.027372 \\ 6 & 0.463047 & -2.544932 & -3.595307 \\ 1 & -0.287266 & -3.308325 & -3.582631 \\ 1 & 0.956133 & -2.529870 & -4.560366 \\ 1 & -0.051162 & -1.597881 & -3.480479 \\ 6 & 2.439680 & -1.557208 & -2.600106 \\ 1 & 2.910018 & -1.660377 & -3.567131 \\ 1 & 3.234815 & -1.631001 & -1.869002 \\ 1 & 1.908397 & -0.618321 & -2.559722 \\ 6 & 2.009441 & -4.112256 & -2.562418 \\ 1 & 2.978902 & -4.116265 & -2.093880 \\ 1 & 2.147097 & -4.429466 & -3.593548 \\ 6 & 1.147754 & -5.270933 & -1.904570 \\ 1 & 1.020996 & -5.120148 & -0.807986 \\ 1 & 1.938001 & -6.058180 & -1.853068 \\ 15 & -1.375811 & -5.688362 & -1.875061 \\ 8 & -2.240697 & -6.559149 & -2.623595 \\ 8 & -1.890157 & -4.333902 & -1.640999 \\ 8 & -0.853833 & -6.259234 & -0.490813 \\ 8 & 0.034726 & -5.735051 & -2.671683 \\ 6 & -1.347158 & -7.444738 & 0.112355 \\ 1 & -0.607354 & -7.877692 & 0.804194 \\ 1 & -1.630340 & -8.221113 & -0.633534 \\ 1 & -2.149898 & -7.190557 & 0.777711 \\ 1 & 9.026663 & 4.931158 & 3.164801 \\ 6 & 9.084809 & 3.902960 & 2.881383 \\ 1 & 10.123117 & 3.782037 & 2.514104 \\ 1 & 8.422512 & 3.647913 & 2.026916 \\ 6 & 8.892561 & 2.979264 & 4.144406 \\ 1 & 7.808954 & 2.725574 & 4.064792 \\ 1 & 9.039529 & 3.660712 & 5.003143 \\ 6 & 9.732878 & 1.711329 & 4.141765 \\ 1 & 10.756365 & 1.977808 & 3.791819 \\ 1 & 9.319154 & 0.950095 & 3.46247 \\ 6 & 9.713436 & 0.961329 & \end{array}$




\begin{tabular}{|c|c|c|c|}
\hline 1 & 10.197858 & -0.031444 & 5.298198 \\
\hline 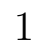 & 8.712141 & 0.722338 & 5.896158 \\
\hline 1 & 10.288546 & 1.464022 & 6.226992 \\
\hline 1 & 5.533345 & -3.156882 & -6.029068 \\
\hline 6 & 5.644481 & -3.298130 & -4.975172 \\
\hline 1 & 4.643930 & -3.287055 & -4.491669 \\
\hline 8 & 6.423721 & -2.145791 & -4.552204 \\
\hline 6 & 6.078617 & -1.695899 & -3.352371 \\
\hline 8 & 5.307175 & -2.139268 & -2.554560 \\
\hline 6 & 6.912947 & -0.418007 & -3.080736 \\
\hline 1 & 6.556871 & -0.013792 & -2.109798 \\
\hline 1 & 6.633460 & 0.314455 & -3.865329 \\
\hline 6 & 6.458508 & -4.589059 & -4.613286 \\
\hline 1 & 7.496375 & -4.409117 & -4.961070 \\
\hline 1 & 6.124535 & -5.441381 & -5.239791 \\
\hline 8 & 6.546281 & -4.764157 & -3.18628 \\
\hline 6 & 5.605493 & -5.439149 & -2.62378 \\
\hline 8 & 4.570682 & -5.785221 & -3.256558 \\
\hline 6 & 5.776424 & -5.789484 & -1.132159 \\
\hline 1 & 6.666797 & -6.447989 & -1.08012 \\
\hline 1 & 4.915478 & -6.301772 & -0.647460 \\
\hline 6 & 8.405085 & -0.667468 & -3.054421 \\
\hline 1 & 9.046618 & 0.238073 & -3.08325 \\
\hline 1 & 8.671083 & -1.273036 & -3.946347 \\
\hline 6 & 8.767030 & -1.501539 & -1.843710 \\
\hline 1 & 8.141869 & -2.421413 & -1.803623 \\
\hline 1 & 8.523624 & -0.952531 & -0.91449 \\
\hline 6 & 10.191952 & -1.934962 & -1.980613 \\
\hline 1 & 10.799588 & -1.013489 & -2.117604 \\
\hline 1 & 10.456209 & -2.580475 & -2.840893 \\
\hline 6 & 10.635272 & -2.767400 & -0.69068 \\
\hline 1 & 9.974643 & -3.641715 & -0.48760 \\
\hline 1 & 10.564640 & -2.029228 & 0.139203 \\
\hline 1 & 11.636814 & -3.135511 & -0.670477 \\
\hline 6 & 6.281150 & -4.660547 & -0.143953 \\
\hline 1 & 5.867150 & -3.670126 & -0.439707 \\
\hline 1 & 7.386489 & -4.660705 & -0.054917 \\
\hline 6 & 5.760664 & -4.880088 & 1.329375 \\
\hline 1 & 4.711889 & -5.232770 & 1.357406 \\
\hline 1 & 5.730943 & -3.971851 & 1.987495 \\
\hline 6 & 6.581573 & -5.877808 & 2.188132 \\
\hline 1 & 7.662168 & -5.672079 & 2.03324 \\
\hline
\end{tabular}




\begin{tabular}{|c|c|c|c|}
\hline 1 & 6.361531 & -6.868421 & 1.744097 \\
\hline 6 & 6.076965 & -5.885102 & 3.633198 \\
\hline 1 & 5.033874 & -6.281001 & 3.651232 \\
\hline 1 & 6.096816 & -4.834650 & 3.964795 \\
\hline 1 & 6.602420 & -6.520274 & 4.317123 \\
\hline 7 & -4.138260 & 3.307577 & -1.043225 \\
\hline 6 & -3.723792 & 4.366939 & -0.065643 \\
\hline 1 & -3.912632 & 5.334338 & -0.513950 \\
\hline 1 & -2.660331 & 4.295244 & 0.028754 \\
\hline 1 & -4.191329 & 4.220930 & 0.899857 \\
\hline 6 & -3.688483 & 1.981820 & -0.577854 \\
\hline 1 & -3.914119 & 1.826002 & 0.467569 \\
\hline 1 & -2.679625 & 1.858190 & -0.950171 \\
\hline 1 & -4.202679 & 1.250178 & -1.188348 \\
\hline 6 & -5.614835 & 3.371639 & -1.239496 \\
\hline 1 & -5.825118 & 2.687947 & -2.044509 \\
\hline 1 & -5.869212 & 4.370145 & -1.579466 \\
\hline 1 & -6.224440 & 3.104352 & -0.390651 \\
\hline 6 & -3.534323 & 3.611710 & -2.414670 \\
\hline 1 & -3.727068 & 2.773133 & -3.060300 \\
\hline 1 & -2.458446 & 3.762129 & -2.393153 \\
\hline 6 & -4.152808 & 4.807340 & -3.173647 \\
\hline 1 & -5.219352 & 4.667036 & -3.453642 \\
\hline 1 & -3.510030 & 4.898383 & -4.081969 \\
\hline 15 & -5.062897 & 7.058798 & -2.100906 \\
\hline 8 & -6.310925 & 6.334635 & -1.740609 \\
\hline 8 & -5.128332 & 8.129971 & -3.147713 \\
\hline 8 & -4.452827 & 7.796947 & -0.80385 \\
\hline 8 & -3.944080 & 6.006735 & -2.438267 \\
\hline 6 & -3.320184 & 8.684311 & -0.894979 \\
\hline 1 & -3.434878 & 9.563805 & -0.220934 \\
\hline 1 & -3.187324 & 8.988461 & -1.954196 \\
\hline 6 & -2.042149 & 8.069917 & -0.453112 \\
\hline 1 & -1.878605 & 7.268451 & -1.196621 \\
\hline 8 & -2.141404 & 7.538067 & 0.889342 \\
\hline 6 & -1.367170 & 6.501597 & 1.108865 \\
\hline 8 & -0.970168 & 5.717839 & 0.261509 \\
\hline 6 & -1.347179 & 6.253142 & 2.594386 \\
\hline 1 & -2.164266 & 5.545432 & 2.828512 \\
\hline 1 & -1.591911 & 7.164234 & 3.177924 \\
\hline 6 & -0.830135 & 9.039996 & -0.536591 \\
\hline 1 & -0.914664 & 9.791409 & 0.26816 \\
\hline
\end{tabular}




$\begin{array}{lccc}1 & -0.703840 & 9.544707 & -1.526295 \\ 8 & 0.468150 & 8.359045 & -0.395656 \\ 6 & 1.016759 & 7.907695 & -1.573498 \\ 8 & 0.679075 & 8.116726 & -2.709137 \\ 6 & 2.368858 & 7.197367 & -1.254966 \\ 1 & 3.155595 & 7.969960 & -1.326707 \\ 1 & 2.390640 & 6.381168 & -2.015389 \\ 6 & 0.043641 & 5.730763 & 2.962680 \\ 1 & 0.740760 & 6.583339 & 3.034366 \\ 1 & 0.430815 & 5.115407 & 2.121945 \\ 6 & 0.123161 & 4.805671 & 4.166209 \\ 1 & -0.563020 & 3.966178 & 3.944159 \\ 1 & -0.220203 & 5.247444 & 5.122052 \\ 6 & 1.545298 & 4.119881 & 4.538524 \\ 1 & 1.933845 & 3.564987 & 3.657262 \\ 1 & 1.298540 & 3.446105 & 5.390367 \\ 1 & 2.167862 & 4.898647 & 4.926902 \\ 6 & 2.469711 & 6.594925 & 0.186350 \\ 1 & 1.582891 & 5.969222 & 0.466433 \\ 1 & 2.591162 & 7.343788 & 0.999217 \\ 6 & 3.665575 & 5.654546 & 0.430273 \\ 1 & 3.490784 & 5.204574 & 1.435844 \\ 1 & 4.524804 & 6.360831 & 0.506342 \\ 6 & 3.830933 & 4.564613 & -0.585604 \\ 1 & 2.827838 & 4.463530 & -1.059082 \\ 1 & 4.138526 & 3.662804 & -0.013217 \\ 6 & 4.989761 & 4.785891 & -1.563435 \\ 1 & 5.621439 & 5.575105 & -1.095009 \\ 1 & 4.755885 & 5.209590 & -2.567766 \\ 6 & 5.963649 & 3.592467 & -1.692199 \\ 1 & 5.384379 & 2.728883 & -2.109414 \\ 1 & 6.306461 & 3.201413 & -0.707030 \\ 1 & 6.826043 & 3.884868 & -2.245804 \\ 8 & 2.076125 & 4.744341 & -4.060794 \\ 1 & 2.144229 & 3.978313 & -3.491354 \\ 1 & 1.157719 & 4.751335 & -4.333158 \\ 8 & -1.330921 & 6.110239 & -3.140326 \\ 1 & -0.856070 & 5.462317 & -2.630115 \\ 1 & -2.251190 & 5.935091 & -2.966460 \\ 8 & 4.262465 & 2.055361 & -47074 \\ 1 & 3.403357 & 2.476171 & \\ 1 & 4.067659 & 1.124729 & \\ & & & \\ 1 & & -4.645857 \\ 1 & \end{array}$




$\begin{array}{cccc}8 & -2.430049 & 0.162435 & -3.740445 \\ 1 & -1.786956 & 0.252075 & -3.042093 \\ 1 & -2.744793 & -0.734925 & -3.661187 \\ 8 & -3.424950 & -1.877546 & -1.949257 \\ 1 & -2.704262 & -1.333343 & -1.611394 \\ 1 & -3.067386 & -2.769251 & -1.952284 \\ 8 & -3.163237 & 2.393568 & -5.119966 \\ 1 & -2.423194 & 2.593271 & -5.702507 \\ 1 & -2.879788 & 1.619636 & -4.633977 \\ 8 & -0.273872 & 3.850079 & -1.931308 \\ 1 & -0.301060 & 2.957842 & -1.570651 \\ 1 & -0.410566 & 4.417021 & -1.178984 \\ 8 & 1.225453 & 1.841055 & -4.157144 \\ 1 & 0.437313 & 2.107217 & -4.635573 \\ 1 & 0.907251 & 1.590197 & -3.293653 \\ 8 & -2.023791 & -3.203054 & 0.616417 \\ 1 & -1.949376 & -2.277448 & 0.398435 \\ 1 & -1.980659 & -3.654914 & -0.226473 \\ 8 & 0.280478 & -4.417668 & 1.475751 \\ 1 & 0.078401 & -5.266360 & 1.088599 \\ 1 & -0.525699 & -3.916954 & 1.393505 \\ 8 & -5.940283 & -1.680071 & -3.032819 \\ 1 & -5.010760 & -1.805760 & -2.821182 \\ 1 & -6.419200 & -2.078809 & -2.301623\end{array}$

